\title{
Intrinsic Limitations in Context Modelling
}

\author{
Maria E. Orłowska \\ Ministry of Science and Higher Education, \\ $1 / 3$ Wspólna Street, \\ 00-529 Warsaw, Poland \\ Phone: (+4822) 629 5703; Fax: (+4822) 5292615
}

\begin{abstract}
Where are the limits in context modelling? Can we ever model context? Do we understand the limits in context modelling, in the context of business process execution?
\end{abstract}

Keywords: context awareness, context-dependent applications, process model, web services, coordination.

The last few years of Information Technology evolution has been strongly influenced by virtually unlimited mobile connectivity between functionally sophisticated hardware devices. Incredible promises of innovative applications, often beyond our imagination, have emerged within pervasive, ubiquitous, and ambient computing, within the semantic web, smart knowledge management, and the management of agile processes, and so on. All have one common characteristic; they rely on a system's ability to observe and capture the application context. Therefore, context awareness, context capturing, context management, de-abstracting context, and linking contexts are the key terms and phrases for these directions in contemporary computer science. The precise meaning of the term "context" depends on the context in which the term is used. This obvious meta-level logical loop often creates confusion and discourages efforts, in different situations, to present a precise semantic specification of this term.

The goal of this presentation is to constructively evaluate the feasibility of expanding the potential for future context-dependent applications in general. We will begin with a short overview of the generic and inherited limitations of current computational models that form the basis of all computing machines we use and most likely will use for many years to come. We will then continue the debate from the perspective of business process modelling issues where the meaning of "context", and dependency of applications on "context", is equally important.

Typically, business process communication is characterized by complex interactions between heterogeneous and autonomous systems within an enterprise, and increasingly between trading partners. Each of the involved parties operates in its own individual context, with different perspectives on the overall process.

Most attempts at business process enforcement can be thought of in terms of two basic approaches. The first "coordinative" paradigm is concerned with the enforcement of a structured, "pre-arranged" process. A complete process

M. Bubak et al. (Eds.): ICCS 2008, Part I, LNCS 5101, pp. 34, 2008.

(C) Springer-Verlag Berlin Heidelberg 2008 
model, reflecting all potential local and global context variations, is designed prior to enacting the process. This approach mainly provides an effective means of coordinating business activities with well-defined dependency relations that can be modelled with simple workflow control constructs such as sequence, choice and fork.

Another approach to process enforcement is concerned with a less structured and a more ad hoc process which is now more commonly quoted since the advent of web services. This is precisely where the use of context specification, discovery or capturing is vital for a realistic chance to deliver any promise. The potential of web service technology in the area of integration and interoperation has generated substantial interest, with initiatives from leading software vendors such as HP, IBM, Microsoft, SAP, Oracle and Sun Microsystems. There is a belief that web services will provide a means for integrating applications, promoting interoperability and facilitating loosely-coupled process management over decentralised environments. A natural question that comes to mind: where is the source of the web services power? Why do integration problems, often semantically and contextually sensitive, become easier to handle under these architectural considerations?

Many extensions to the basic web service functionality have been recently proposed with the aim of capturing more meaningful semantics and contexts than simply service invocations, enabling the modelling and implementation of business processes in the web service context. This coordination layer is said to provide web service 'orchestration', 'choreography' or 'harmonization'.

Even a superficial analysis of the potential obstacles with deployment, either through coordinative or collaborative approaches for cross-organizational business process support, reveals that the main issues are related to context modelling of the loosely coupled application environments such that global goals are tractably achieved.

This talk will highlight the issues outlined above with an attempt to provide well-defined feasibility limits for such context sensitive loosely coupled applications. 